\title{
Hyperglycemia, inflammatory response and infarct size in obstructive acute myocardial infarction and MINOCA
}

\author{
Pasquale Paolisso ${ }^{1 \dagger}$, Alberto Foà ${ }^{1 \dagger}$, Luca Bergamaschi ${ }^{1}$, Francesco Donati ${ }^{1}$, Michele Fabrizio ${ }^{1}$, \\ Chiara Chiti ${ }^{1}$, Francesco Angeli', Sebastiano Toniolo ${ }^{1}$, Andrea Stefanizzi ${ }^{1}$, Matteo Armillotta ${ }^{1}$, Paola Rucci², \\ Gianmarco lannopollo ${ }^{3}$, Gianni Casella ${ }^{3}$, Cinzia Marrozzini ${ }^{1}$, Nazzareno Galiè ${ }^{1}$ and Carmine Pizzi ${ }^{* *}$
}

\begin{abstract}
Background: Hyperglycemia has been associated with increased inflammatory indexes and larger infarct sizes in patients with obstructive acute myocardial infarction (obs-AMI). In contrast, no studies have explored these correlations in non-obstructive acute myocardial infarction (MINOCA). We investigated the relationship between hyperglycemia, inflammation and infarct size in a cohort of AMI patients that included MINOCA.

Methods: Patients with AMI undergoing coronary angiography between 2016 and 2020 were enrolled. The following inflammatory markers were evaluated: C-reactive protein, neutrophil-to-lymphocyte ratio (NLR), platelet-to-lymphocyte ratio (PLR) and neutrophil-to-platelet ratio (NPR). Myocardial infarct size was measured by peak high sensitivity troponin I (Hs-Tnl) levels, left-ventricular-end-diastolic-volume (LVEDV) and left ventricular ejection fraction (LVEF).

Results: The final study population consisted of 2450 patients with obs-AMI and 239 with MINOCA. Hyperglycemia was more prevalent among obs-AMI cases. In all hyperglycemic patients—obs-AMI and MINOCA—NLR, NPR, and LPR were markedly altered. Hyperglycemic obs-AMI subjects exhibited a higher Hs-Tnl $(p<0.001)$, a larger LVEDV $(p=0.003)$ and a lower LVEF $(p<0.001)$ compared to normoglycemic ones. Conversely, MINOCA patients showed a trivial myocardial damage, irrespective of admission glucose levels.
\end{abstract}

Conclusions: Our data confirm the association of hyperglycemic obs-AMI with elevated inflammatory markers and larger infarct sizes. MINOCA patients exhibited modest myocardial damage, regardless of admission glucose levels.

Keywords: Hyperglycemia, Inflammation, Infarct size, MINOCA, Obstructive acute myocardial infarction

\section{Background}

Hyperglycemia frequently occurs in patients admitted for acute myocardial infarction (AMI), irrespective of a previously documented diabetes mellitus (DM) [1]. In particular, approximately $10 \%$ to $20 \%$ of non-diabetic

\footnotetext{
*Correspondence: carmine.pizzi@unibo.it

${ }^{\dagger}$ Pasquale Paolisso and Alberto Foà contributed equally to this work ${ }^{1}$ Unit of Cardiology, Department of Experimental, Diagnostic and Specialty Medicine-DIMES, University of Bologna, Via Giuseppe Massarenti 9, Bologna 40138, Italy

Full list of author information is available at the end of the article
}

AMI patients have significant hyperglycemia [2]. Recent data demonstrated that hyperglycemia is associated with an increased risk of major adverse cardiovascular events (MACE) [2, 3]. Additionally, amongst patients with large infarct sizes, hyperglycemia has been identified as a prognostic marker both in patients with and without diabetes [4-6].

So far, it is unexplained whether elevated admission high glucose levels (aHGL) are a marker of more extensive myocardial damage or a prognostic risk factor in patients with AMI [7].

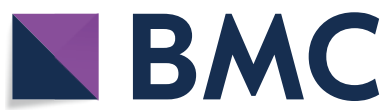

c The Author(s) 2021. This article is licensed under a Creative Commons Attribution 4.0 International License, which permits use, sharing, adaptation, distribution and reproduction in any medium or format, as long as you give appropriate credit to the original author(s) and the source, provide a link to the Creative Commons licence, and indicate if changes were made. The images or other third party material in this article are included in the article's Creative Commons licence, unless indicated otherwise in a credit line to the material. If material is not included in the article's Creative Commons licence and your intended use is not permitted by statutory regulation or exceeds the permitted use, you will need to obtain permission directly from the copyright holder. To view a copy of this licence, visit http://creativeco mmons.org/licenses/by/4.0/. The Creative Commons Public Domain Dedication waiver (http://creativecommons.org/publicdomain/ zero/1.0/) applies to the data made available in this article, unless otherwise stated in a credit line to the data. 
In order to unravel the association between aHGL and the increased risk of adverse cardiovascular events, several potential explanations have been suggested. Systemic immune activation, modification of platelet function and thrombotic-fibrinolysis system, abnormal autonomic tone, increased oxidative stress, endothelial dysfunction and impaired myocardial contractility seem to play a role in myocardial damage [8-10].

Etiopathogenetic mechanisms underlying hyperglycemia in the acute phase of myocardial infarction have not been fully elucidated. Blood glucose levels can be transiently elevated either as a stress response to acute illness (stress hyperglycemia), resulting from an inflammatory and adrenergic adaptation to ischemic injury (release of catecholamines and steroids and glycogenolysis induction), or as a reflection of an underlying abnormal glucometabolic state.

In the context of AMI, a series of ischemia-mediated pathophysiological events occur, generating an intense inflammatory response. Neutrophils are the first leukocytes detected in infarcted areas, followed by monocytes and lymphocytes, which, releasing proteo-enzymes and cytokines, phagocytize necrotic debris and promote the subsequent proliferative process [11]. Additionally, activated platelets, besides acutely precipitating vascular obstruction, further amplify the inflammatory response interacting with neutrophils, monocytes and lymphocytes. Therefore, the role of inflammatory cells is not limited to the acute ischemic event but drives the chronic atherosclerotic process as well.

Recent accumulating evidence suggests that neutrophil-to-lymphocyte ratio (NLR), platelet-to-lymphocyte ratio (PLR) and neutrophil-to-platelet ratio (NPR) might be considered as biomarkers of systemic inflammation and have been associated with poor clinical outcomes in various cardiovascular diseases, including acute coronary syndromes [12-15].

The link between aHGL and inflammation is nowadays well established as it is the prognostic role of hyperglycemia in the context of AMI with obstructive coronary artery disease (obs-AMI). On the other hand, the relationship between hyperglycemia and inflammatory response in myocardial infarction with non-obstructive coronary arteries (MINOCA) is still poorly explored.

Our study sought to investigate the association between hyperglycemia and inflammatory status as well as myocardial damage/severity in patients with obs-AMI versus MINOCA.

\section{Materials and methods Patients}

All consecutive patients hospitalized for AMI (Policlinico Sant'Orsola-Malpighi Hospital and Maggiore Hospital,
Bologna-Italy) who underwent coronary angiography (CAG) within the first $72 \mathrm{~h}$ from admission between January 2016 and March 2020 were included in the study. AMI was diagnosed in the presence of an increase and/ or decrease of cardiac biomarker (troponin I high sensitivity-Tn I Hs) with at least one value above the 99th percentile upper reference limit associated with one of the following: symptoms of ischemia, new or presumed new significant ST-segment-T wave changes or new left bundle branch block, development of pathological $Q$ waves in the EKG, and imaging evidence of new loss of viable myocardium or new regional wall motion abnormality [16, 17]. MINOCA was diagnosed according to the 2016 ESC MINOCA Position Paper criteria [18, 19]. Patients whose admission glycemia was not available were excluded from the study. Other exclusion criteria were severe valvular heart disease, prosthetic heart valves, severe anaemia, major acute bleeding, pulmonary embolism, fever $\left(38{ }^{\circ} \mathrm{C}\right)$, hypertensive crisis, chronic renal failure (glomerular filtration rate $<30 \mathrm{~mL} / \mathrm{min} / 1.73$ $\mathrm{m} 2$ ), autoimmune diseases, malignancies or ongoing cardiotoxic medications, and congenital heart disease.

Data were collected as part of an approved multicenter observational study called "AMIPE: Acute Myocardial Infarction, Prognostic and Therapeutic Evaluation" (ClinicalTrials.gov Identifier: NCT03883711). The present study was conducted according to the principles of the Declaration of Helsinki; all patients were informed about their participation in the registry and provided informed consent for the anonymous publication of scientific data.

\section{Inflammatory biomarkers and infarct size detection}

The inflammatory response was evaluated using the following parameters: NLR, NPR, PLR, C-Reactive Proteine. In particular, NLR is the ratio of neutrophil and lymphocyte counts, NPR is the ratio of neutrophil and platelet counts, and PLR is obtained by dividing the platelet count by the lymphocytes. The other laboratory parameters were determined according to standard protocols.

For all patients, blood for hs-TnI evaluation was drawn at the moment of hospital admission and every 3-6 h thereafter for the following $24 \mathrm{~h}$. The hs-TnI peak was considered the highest value before its fall. Comprehensive echocardiographic studies, including Doppler studies, were performed according to the current European recommendations [20].

All patients underwent 2D-echocardiogram before discharge, approximately 3 days after hospitalization. All studies were performed by experienced operators using Philips EPIQ and Affiniti ultrasonography machines. At least 3 consecutive beats were recorded for each view and all images were stored for offline analysis. Left ventricular ejection fraction (LVEF) was calculated with the biplane 
Simpson's method acquiring volumes in both 4- and 2 -chamber views, according to the European Association of Cardiovascular Imaging Guidelines [20]. Myocardial infarct size was also estimated using the left ventricular end-diastolic volume (LVEDV) and the left ventricular ejection fraction (LVEF).

\section{Blood glucose and definition of hyperglycemia}

Blood glucose levels were assessed at admission as part of the standard evaluation. Pre-existing DM was defined as known DM at the time of hospitalization irrespective of the therapeutic management (either diet and lifestyle measures alone or additional administration of oral glucose-lowering medication and insulin) [21]. According to the American Heart Association Scientific Statement, patients were categorised based on admission glucose levels as follows: normoglycemia $<140 \mathrm{mg} / \mathrm{dl}$ and hyperglycemia $\geq 140 \mathrm{mg} / \mathrm{dl}[2]$.

\section{Statistical analysis}

We analyzed the correlation of inflammatory and infarct size markers with hyperglycemia at hospital admission in patients with obs-AMI and in those with MINOCA. To this purpose, we first assessed the distribution of laboratory parameters using Shapiro-Wilks test and the homogeneity of variance using Levene's test. We then compared laboratory parameters and infarct sizes between patients with or without hyperglycemia using Mann-Whitney U test or Student's t-test as appropriate. Categorical variables were compared between groups using $X$ [2] test. Lastly, we investigated differences in inflammatory biomarkers and infarct size of hyperglycemia in obstructive-AMI (obs-AMI) and MINOCA patients using univariate logistic regression models. The significance level was set to $\mathrm{p}<0.05$, and all analyses were performed using Stata 13.1 (Stata Corp., College Station, Texas, 2013) and IBM SPSS, version 25.0 (Fig. 1).

\section{Results}

A total of 2795 patients with suspected AMI who underwent coronary angiography within $72 \mathrm{~h}$ of symptom onset were evaluated. Following diagnostic workup, 94 patients were excluded from the study due to a non-ischemic troponin elevation, including 16 myocarditis and 52 Takotsubo syndromes. Among cases diagnosed with AMI, 12 patients (10 obs-AMI and 2 MINOCA) were excluded because blood glucose level at hospital admission was not available. The final study population consisted of 2450 patients with obs-AMI and 239 with MINOCA. Clinical and angiographic characteristics of obs-AMI subjects as well as etiopathological causes of MINOCA are shown in Table 1. Both groups were divided according to the presence of aHGL. Demographic and clinical characteristics are shown in Table 2. Overall, admission aHGL was noticed in 1017 patients (37.8\%), more frequently in patients with obs-AMI compared to MINOCA ( $40 \%$ versus $16.7 \% ; \mathrm{p}<0.001$ ). The parameters of infarct size and myocardial damage/inflammation of each group are presented in Table 3 and Fig. 2.

\section{Obstructive-AMI: hyperglycemic vs normoglycemic patients}

Over the 2450 patients with obs-AMI, hyperglycemia at admission was detected in 977 (40\%) while no cases of hypoglycemia were observed. Notably, among hyperglycemic patients, a known T2DM was recorded in approximately half cases while less than $10 \%$ of normoglycemic subjects were diabetic. Hyperglycemic patients exhibited a worse cardiovascular risk profile and more comorbidities compared to normoglycemic ones. In fact, they were older, generally overweight, with a higher prevalence of hypertension and a history of cardiovascular events. As expected, a hyperglycemic status reflected an underlying altered glycol-lipid profile and was associated with a greater comorbidity burden, such as atrial fibrillation and chronic lung disease. Over $90 \%$ of normoglycemic obs-AMI patients presented with typical angina, while the percentage dropped to $83 \%$ among hyperglycemic patients $(\mathrm{p}<0.001)$. Lastly, STEMI diagnosis at admission was similar between subgroups.

\section{MINOCA: hyperglycemic vs normoglycemic patients}

Among the 239 patients diagnosed with MINOCA, only $16.7 \%$ exhibited a hyperglycemic state at admission, and no cases of hypoglycemia were observed. Hyperglycemic patients were significantly older, with a higher prevalence of hypertension. Similarly to the obstructive cohort, hyperglycemic cases showed a worse metabolic profile, with higher cholesterol levels and a greater prevalence of T2DM. Interestingly, the glycemic status did not affect the history of cardiovascular events or the prevalence of comorbidities, except for atrial fibrillation which was more frequent among hyperglycemic patients. Again, typical angina was frequently observed among normoglycemic patients, while $35 \%$ of hyperglycemic subjects had a different clinical presentation $(\mathrm{p}=0.004)$. STEMI was equally diagnosed among cohorts.

\section{Impact of admission hyperglycemia on inflammatory markers and infarct size: obstructive-AMI vs MINOCA patients}

In obs-AMI patients, total white blood cell count, neutrophils, platelets, CRP and peak troponin I levels were significantly higher in aHGL group compared to normoglycemic cases (Table 3). Moreover, all inflammatory parameters (NLR, NPR and LPR) were markedly altered 


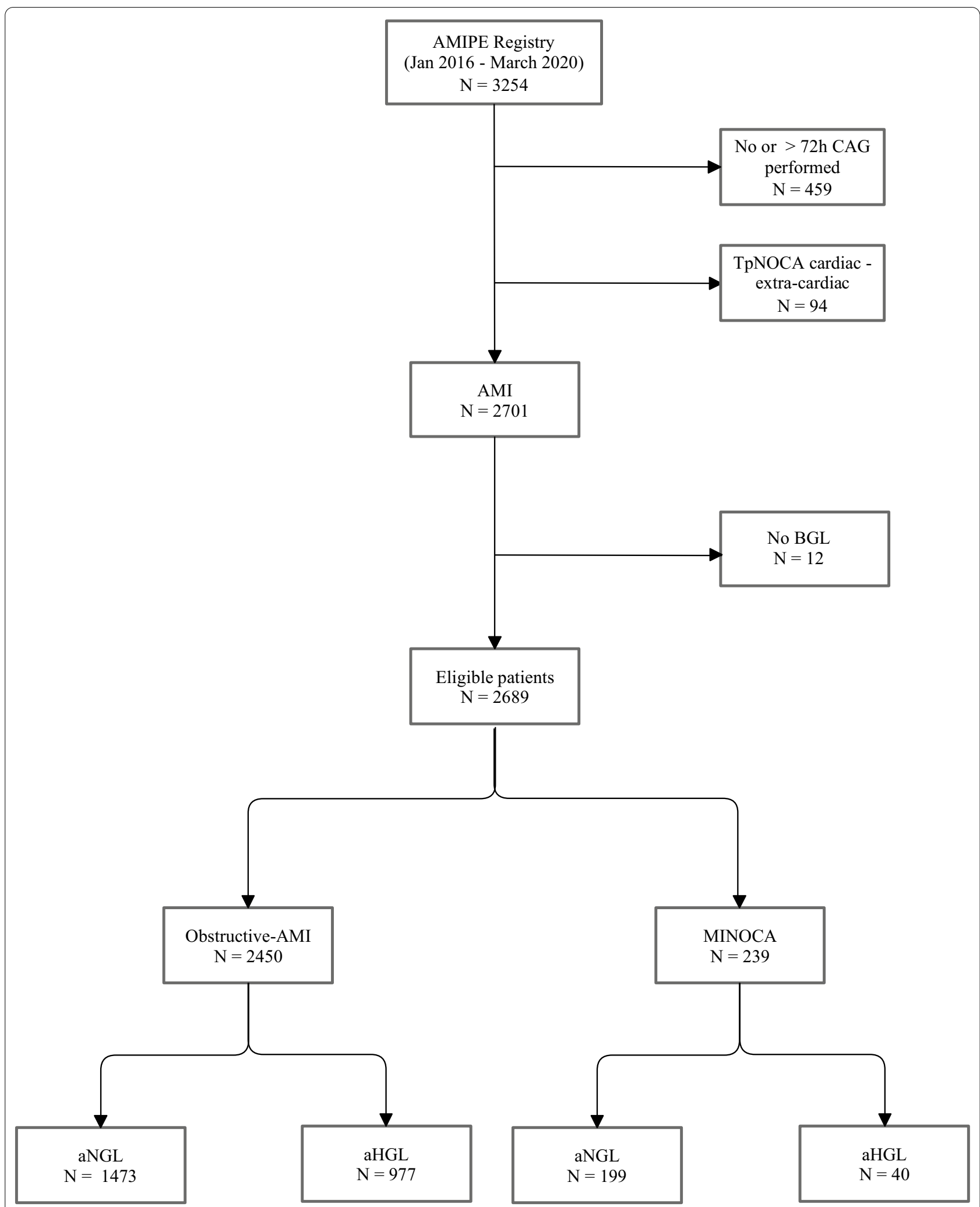

Fig. 1 Flow chart Study. CAG coronary angiography, AMI acute myocardial infarction, TpNOCA troponin-positive non-obstructive coronary arteries, Obs-AMI obstructive myocardial infarction, MINOCA myocardial infarction with non-obstructive coronary arteries, aBGL admission blood glucose level, aHGL admission high glucose level, aNGL admission normal glucose level 
Table 1 Clinical and angiographic characteristics of obstructive-AMI and MINOCA patients

\begin{tabular}{|c|c|c|c|c|c|}
\hline & \multicolumn{2}{|c|}{ Obstructive AMI N=2450 } & \multicolumn{3}{|l|}{ MINOCA N $=239$} \\
\hline & STEMI N=1116 & NSTEMI N = 1334 & & STEMI $\mathbf{N}=28$ & NSTEMI N $=211$ \\
\hline Lesion Location & & & Causes & & \\
\hline LM lesion, n (\%) & $47(4.2)$ & $195(14.6)$ & Epicardial coronary spasm & $1(3.6)$ & $13(6.2)$ \\
\hline LAD lesion, n (\%) & $954(85.4)$ & $988(74)$ & SCAD & $9(32.1)$ & $26(12.3)$ \\
\hline LCx lesion, n (\%) & $412(36.9)$ & $520(38.9)$ & Coronary embolism & $4(14.3)$ & $0(0)$ \\
\hline \multirow[t]{2}{*}{ RC lesion, $\mathrm{n}(\%)$} & $744(66.7)$ & $759(56.9)$ & Atherosclerotic plaque disruption (type I) & $5(17.9)$ & $31(14.7)$ \\
\hline & & & Supply-demand mismatch (type II) & $9(32.1)$ & $141(66.8)$ \\
\hline Number of Vessels & & & Number of vessels with stenosis & & \\
\hline LM, n (\%) & $37(3.3)$ & $148(11.1)$ & LM (1-20\%), n (\%) & $4(14.3)$ & $1(0.5)$ \\
\hline 1 Vessel, n (\%) & $667(59.8)$ & $459(34.4)$ & 1 Vessel (20-49\%), n (\%) & $3(10.7)$ & $31(14.6)$ \\
\hline 2 Vessels, n (\%) & $303(27.2)$ & $383(28.7)$ & 2 Vessels (20-49\%), n (\%) & $3(10.7)$ & $13(6.2)$ \\
\hline 3 Vessels, n (\%) & $109(9.7)$ & $344(25.8)$ & 3 Vessels (20-49\%), n (\%) & $0(0)$ & $3(1.4)$ \\
\hline
\end{tabular}

AMI Acute Myocardial Infarction, LAD Left Anterior Descending artery, LCX Left Circumflex, LM Left Main, MINOCA myocardial Infarction with Non-Obstructive Coronary Arteries, NSTEMI Non-ST-segment elevation myocardial infarction, RC Right Coronary artery, STEMI ST-segment elevation myocardial infarction, SCAD Spontaneous coronary artery dissection

in hyperglycemic subjects, both at admission and after $24 \mathrm{~h}$ (Fig. 2 and Table 3). Additionally, these patients exhibited a greater LVEDV and a lower LVEF compared to normoglycemic ones. In the MINOCA cohort, inflammatory markers at admission were significantly higher in aHGL group compared to normoglycemic patients while no differences were observed after 24 h. Importantly, hyperglycemic and normoglycemic subjects exhibited similar infarct sizes (Table 3).

Comparing hyperglycemic obs-AMI and hyperglycemic MINOCA patients, similar values of inflammatory parameters were detected at admission. In contrast, higher levels of WBC and neutrophils were evident after $24 \mathrm{~h}$ among the obs-AMI cohort. Notably, hyperglycemic obs-AMI subjects exhibited higher troponin levels, greater LVEDVs and a depressed LV function, all markers of larger infarct size (Table 3).

\section{Discussion}

Our study was focused on the interplay between hyperglycemia, inflammation and infarct size in a cohort of patients admitted with acute myocardial infarction, including cases of MINOCA, a still poorly investigated nosological entity.

Hyperglycemia was homogeneously associated with an increase of all inflammatory indices at admission, irrespective of the underlying ischemic pathophysiological mechanism, either obs-AMI or MINOCA. Importantly, hyperglycemia correlated with the detection of large infarct sizes in patients with obs-AMI while no differences were observed between normoglycemic and hyperglycemic MINOCA cases, which exhibited a modest myocardial damage.

\section{Hyperglycemia and inflammation markers in obstructive-AMI}

Among our overall study population, hyperglycemia was more frequently observed in patients with obs-AMI. This subgroup of hyperglycemic subjects exhibited an "inflammatory status" as expressed by increased levels of all measured inflammatory markers. High values of NLR, NPR, PLR and CRP had been previously described in this setting, and our results are in line with the existing literature, confirming the relationship between glycemic disorders and inflammation in the context of obs-AMI $[22,23]$. Indeed, the activation of inflammatory mediators and pathways is vastly described as a cornerstone of atherosclerosis [24], not only in terms of chronic arterial remodelling but also favouring plaque instability and rupture [25]. Moreover, some studies have identified an association of elevated inflammatory markers, including NPR and NLR, with larger infarct sizes and an increased risk of short-term mortality [12, 13, 26].

Hyperglycemia-mediated alterations may further precipitate the atherosclerotic process [27-29]. In fact, not only does hyperglycemia amplify the inflammatory cascade, but it is also promoted by the inflammatory process itself throughout the generation of insulin-resistance and gluconeogenesis [30-32]. As a result, the interplay between hyperglycemia and inflammation triggers a vicious circle, ultimately leading to a heightened atherosclerotic burden and plaque rupture [33] with an increased mortality risk [1, 23, 34].

\section{Hyperglycemia and inflammatory markers in MINOCA}

The main novelty of our study is that for the first time, we investigated the correlation between glycemic levels 
Table 2 Demographic, clinical, laboratory findings and treatment of obstructive-AMI and MINOCA patients, according to admission to hyperglycemia

\begin{tabular}{|c|c|c|c|c|c|c|}
\hline & \multicolumn{2}{|c|}{$\begin{array}{l}\text { Obstructive-AMI } \\
\mathrm{N}=2450\end{array}$} & \multirow[b]{2}{*}{$p$-value } & \multicolumn{2}{|l|}{$\begin{array}{l}\text { MINOCA } \\
\mathrm{N}=239\end{array}$} & \multirow[b]{2}{*}{$p$-value } \\
\hline & $\begin{array}{l}\mathrm{aHGL} \\
\mathrm{N}=977\end{array}$ & $\begin{array}{l}\text { aNGL } \\
N=1473\end{array}$ & & $\begin{array}{l}\mathrm{aHGL} \\
\mathrm{N}=40\end{array}$ & $\begin{array}{l}\text { aNGL } \\
\mathrm{N}=199\end{array}$ & \\
\hline Age, years, median (IQR) & $72.0(62.0-80.0)$ & $68.0(58.0-78.0)$ & $<0.001$ & $74(67-81)$ & $68(53-77)$ & 0.001 \\
\hline Gender Female, n (\%) & $280(28.7)$ & $383(26)$ & 0.1 & $28(70)$ & $129(64.8)$ & 0.5 \\
\hline BMI Kg/m², median (IQR) & $26.8(24.2-30.3)$ & $26.2(23.9-29.0)$ & 0.001 & $25.9(22.8-29.2)$ & $25.6(22.4-28.2)$ & 0.6 \\
\hline \multicolumn{7}{|l|}{ Cardiovascular risk factors } \\
\hline Current/past smoking, n (\%) & $547(56.3)$ & $908(62.4)$ & 0.007 & $13(32.5)$ & $88(44.7)$ & 0.1 \\
\hline Hypertension, n (\%) & $720(74.2)$ & $967(65.9)$ & $<0.001$ & $30(75)$ & $129(65.2)$ & 0.2 \\
\hline Dyslipidemia, n (\%) & $595(61.3)$ & $898(61.2)$ & 0.9 & $21(52.5)$ & $123(61.8)$ & 0.3 \\
\hline Type-2 diabetes, n (\%) & $477(48.8)$ & $113(7.7)$ & $<0.001$ & $12(30.0)$ & $11(5.5)$ & $<0.001$ \\
\hline \multicolumn{7}{|l|}{ Medical history } \\
\hline Previous AMI, n (\%) & $238(24.5)$ & $290(19.8)$ & 0.006 & $2(5.4)$ & $18(9.8)$ & 0.4 \\
\hline Previous stroke, n (\%) & $80(8.2)$ & $79(5.4)$ & 0.005 & $2(5.0)$ & $11(5.5)$ & 0.8 \\
\hline COPD, n (\%) & $122(12.5)$ & $152(10.3)$ & 0.09 & $5(12.5)$ & $21(10.6)$ & 0.7 \\
\hline PAD, n (\%) & $103(10.6)$ & $85(5.8)$ & $<0.001$ & $2(5)$ & $5(2.5)$ & 0.4 \\
\hline \multicolumn{7}{|l|}{ Clinical presentations } \\
\hline Angina, $\mathrm{n}(\%)$ & $813(83.7)$ & $1337(91)$ & $<0.001$ & $28(70)$ & $170(85.4)$ & 0.02 \\
\hline $\mathrm{HR}$, median (IQR) & $81(70-97)$ & $75(65-88)$ & $<0.001$ & $95(76-134)$ & $80(66-93)$ & $<0.001$ \\
\hline SBP, median (IQR) & $140(120-160)$ & $140(120-160)$ & 0.5 & $140(118-160)$ & $140(120-155)$ & 0.7 \\
\hline DBP, median (IQR) & $80(70-90)$ & $80(70-90)$ & 0.3 & $80(70-85)$ & $80(70-90)$ & 0.4 \\
\hline Atrial fibrillation, n (\%) & $103(10.6)$ & $93(6.4)$ & $<0.001$ & $13(32.5)$ & $14(7.1)$ & $<0.001$ \\
\hline STEMI, n (\%) & $468(47.9)$ & $648(43.9)$ & 0.057 & $5(12.5)$ & $23(11.6)$ & 0.8 \\
\hline \multicolumn{7}{|l|}{ Laboratory parameters } \\
\hline Hemoglobin g/dL, median (IQR) & $13.6(12.1-15.0)$ & $14.0(12.7-15.1)$ & 0.001 & $13.2(12.1-14.8)$ & $13.4(12.1-14.5)$ & 0.9 \\
\hline Admission BGL level mg/dL, median (IQR) & $183(157-238)$ & $111(99-122)$ & $<0.001$ & $183(154-227)$ & $104(93-117)$ & $<0.001$ \\
\hline Discharge BGL level, mg/dl, median (IQR) & $114(97-145)$ & $98(85-112)$ & $<0.001$ & $105(92-127)$ & $97(85.0-111)$ & 0.02 \\
\hline $\mathrm{HbA1c}$, mmol/mol, median (IQR) & $47(40-60)$ & $37(34-40)$ & $<0.001$ & $40(37-50)$ & $36(32-40)$ & 0.003 \\
\hline Creatinine mg/dl, median (IQR) & $1.0(0.9-1.3)$ & $0.9(0.8-1.1)$ & $<0.001$ & $1.0(0.7-1.2)$ & $0.8(0.7-1.0)$ & 0.04 \\
\hline C-TOT, mg/dL median (IQR) & $181(149-216)$ & $192(161-222)$ & $<0.001$ & $169(151-205)$ & $197(167-224)$ & 0.03 \\
\hline C-LDL, mg/dL median (IQR) & $111(85-139)$ & $121(93-149)$ & $<0.001$ & $97(84-127)$ & $118(97-144)$ & 0.04 \\
\hline Tryglicerides, median (IQR) & $116(84-165)$ & $112(83-153)$ & 0.02 & $116(89-143)$ & $111(80-153)$ & 0.8 \\
\hline \multicolumn{7}{|l|}{ Admission medical therapy } \\
\hline Aspirin, n (\%) & $374(38.6)$ & $501(34.2)$ & 0.03 & $8(20)$ & $50(25.1)$ & 0.5 \\
\hline P2Y12 Inhibitor,s n (\%) & $99(10.2)$ & $110(7.5)$ & 0.02 & $2(5)$ & $9(4.5)$ & 0.9 \\
\hline Beta-blockers, n (\%) & $401(41.4)$ & $520(35.6)$ & 0.004 & $17(42.5)$ & $60(30.2)$ & 0.1 \\
\hline RAAS inhibitors, n (\%) & $504(52)$ & $659(45.1)$ & 0.002 & $23(57.5)$ & $64(32.2)$ & 0.002 \\
\hline Statins, n (\%) & $297(30.7)$ & $395(27)$ & 0.048 & $15(37.5)$ & $51(25.6)$ & 0.1 \\
\hline \multicolumn{7}{|l|}{ Admission glucose-lowering agents } \\
\hline Insulin sensitizers (metformin), n (\%) & $259(31.4)$ & $69(4.3)$ & $<0.001$ & $5(14.7)$ & $8(4)$ & 0.01 \\
\hline Insulin providers (sulfonylureas), n (\%) & $160(19.4)$ & $37(2.3)$ & $<0.001$ & $3(8.8)$ & $2(1.0)$ & 0.004 \\
\hline DPP-4 Inhibitors, n (\%) & $29(3.5)$ & $6(0.4)$ & $<0.001$ & $1(2.9)$ & $1(0.5)$ & 0.1 \\
\hline GLP-1 Agonist, n (\%) & $7(0.8)$ & $2(0.1)$ & 0.02 & 0 & 0 & 0.99 \\
\hline SGLT-2 Inhibitors, n (\%) & $3(0.4)$ & $2(0.1)$ & 0.06 & 0 & 0 & 0.99 \\
\hline Insulin, n (\%) & $120(14.6)$ & $27(1.7)$ & $<0.001$ & $2(5.9)$ & 0 & 0.001 \\
\hline PCl total, n (\%) & $809(83)$ & 1213 (82.8) & 0.9 & 0 & 0 & 0.99 \\
\hline PCI NSTEMI, n (\%) & 325 (75.9) & $586(76.4)$ & 0.85 & 0 & 0 & 0.99 \\
\hline
\end{tabular}

Continuous variables are presented as median (IQR) while categorical ones as $\mathrm{n}(\%)$. AMI acute myocardial infarction, MINOCA myocardial infarction with nonobstructive coronary arteries, Obs-AMI obstructive acute myocardial infarction, aHGL admission High Glucose Level, aNGL admission normal glucose level, BMI body max index, COPD chronic obstructive pulmonary disease, HR heart rate, SBP systolic blood pressure, DBP diastolic blood pressure, STEMI ST-segment Elevation Myocardial Infarction, BGL blood glucose level, HbA1c glycated hemoglobin, C-TOT = total cholesterol, LDL-c LDL cholesterol, RAAS Renin-angiotensin-aldosterone system, DPP-4 dipeptidyl peptidase 4, GLP-1 glucagon-like peptide 1, SGLT-2 Sodium glucose co-transporter 2, PCI percutaneous coronary intervention The last column shows the comparison between hyperglycemic obstructive-AMI and MINOCA patients 
Table 3 Inflammation markers and infarct size in Obstructive-AMI and MINOCA patients, according to admission hyperglycemia

\begin{tabular}{|c|c|c|c|c|c|c|c|}
\hline & \multicolumn{3}{|l|}{$\begin{array}{l}\text { Obstructive-AMI } \\
\mathrm{N}=2450\end{array}$} & \multicolumn{3}{|l|}{$\begin{array}{l}\text { MINOCA } \\
\mathrm{N}=239\end{array}$} & \multirow{2}{*}{$\begin{array}{l}\text { aHGL } \\
\text { Obs-AMI vs } \\
\text { MINOCA } \\
p \text {-value }\end{array}$} \\
\hline & $\begin{array}{l}\text { aHGL } \\
\mathrm{N}=977\end{array}$ & $\begin{array}{l}\text { aNGL } \\
\mathrm{N}=1473\end{array}$ & $p$-value & $\begin{array}{l}\text { aHGL } \\
\mathrm{N}=40\end{array}$ & $\begin{array}{l}\text { aNGL } \\
N=199\end{array}$ & $p$-value & \\
\hline \multicolumn{8}{|c|}{ Inflammation markers (admission_-T0) } \\
\hline WBC N/Ml, median (IQR) & $10.5(8.1-13.3)$ & $9.2(7.4-11.6)$ & $<0.001$ & $10.4(8.1-14.9)$ & $8.1(6.6-10.1)$ & $<0.001$ & ns \\
\hline 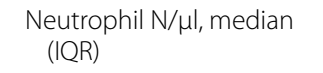 & $7197(5385-10,249)$ & $6228(4678-8623)$ & $<0.001$ & $7933(5637-11,443)$ & $5305(4053-7361)$ & $<0.001$ & ns \\
\hline $\begin{array}{l}\text { Lymphocyte N/Ml, median } \\
\text { (IQR) }\end{array}$ & $1710(1187-2530)$ & $1787(1312-2496)$ & 0.2 & $1736(1165-2164)$ & $1840(1385-2310)$ & 0.3 & ns \\
\hline $\begin{array}{l}\text { PLTs count } \times 10^{9} \text { per } \mathrm{L} \text {, } \\
\text { median (IQR) }\end{array}$ & $233(193-282)$ & $228(189-275)$ & 0.1 & $234(195-287)$ & $239(200-289)$ & 0.8 & ns \\
\hline CRP mg/dL, median (IQR) & $0.5(0.2-1.4)$ & $0.4(0.2-0.8)$ & $<0.001$ & $0.5(0.2-1.6)$ & $0.3(0.1-0.7)$ & 0.04 & ns \\
\hline \multicolumn{8}{|c|}{ Inflammation markers (24 h-T1) } \\
\hline WBC N/Ml, median (IQR) & $9.7(7.9-12.3)$ & $8.7(7.0-10.9)$ & $<0.001$ & $7.9(7.0-10.9)$ & $7.4(6.2-8.8)$ & 0.04 & 0.008 \\
\hline 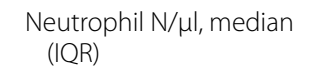 & 6935 (5344-9392) & $5881(4425-7730)$ & $<0.001$ & $5505(3851-9122)$ & $4590(3519-6527)$ & 0.08 & 0.026 \\
\hline $\begin{array}{l}\text { Lymphocyte N/Hl, median } \\
\text { (IQR) }\end{array}$ & $1688(1187-2219)$ & $1839(1376-2407)$ & $<0.001$ & $2012(1042-2419)$ & $1817(1367-2304)$ & 0.9 & ns \\
\hline CRP mg/dL, median (IQR) & $1.1(0.4-4.3)$ & $0.7(0.3-1.8)$ & $<0.001$ & $1.0(0.4-2.3)$ & $0.5(0.2-1.0)$ & 0.06 & ns \\
\hline \multicolumn{8}{|l|}{ Infarct size } \\
\hline LVEDV ml, median (IQR) & $108(84-135)$ & $100(83-121)$ & 0.003 & $80(70-121)$ & $89(74-107)$ & 0.7 & 0.016 \\
\hline LV EF \%, median (IQR) & $47(40-56)$ & $55(45-60)$ & $<0.001$ & $59(50-61)$ & $60(53-62)$ & 0.8 & $<0.001$ \\
\hline $\begin{array}{l}\text { Peak hs Troponin ng/L, } \\
\text { median (IQR) }\end{array}$ & $6556(959-35,531)$ & $2936(576-18,164)$ & $<0.001$ & $369(133-901)$ & $461(113-1661)$ & 0.5 & $<0.001$ \\
\hline
\end{tabular}

Continuous variables are presented as median (IQR) while categorical ones as $\mathrm{n}$ (\%).AMI Acute myocardial infarction, MINOCA Myocardial infarction with nonobstructive coronary arteries, Obs-AMI Obstructive acute myocardial infarction, $a H G L$ Admission high glucose level, aNGL Admission normal glucose level, WBC White blood cell, PLTS Platelets, NLR Neutrophil-to-lymphocyte ratio, PLR Platelet-to-lymphocyte ratio, NPR Neutrophil-to-platelet ratio, CRP C-reactive protein, LVEDV Left ventricular end diastolic diameter; LVEDV Left ventricular end diastolic volume, LVEF Left ventricular ejection fraction, $H s$ High sensitivity

inflammatory markers in MINOCA patients. Similarly, to the results observed in obs-AMI, hyperglycemic MINOCA subjects had higher values of NLR, NPR, and PLR than normoglycemic ones.

Shared underlying pathophysiological mechanisms may explain the complex interplay between hyperglycemia, inflammation and MINOCA. In particular, a central role seems to be played by endothelial dysfunction [35]. In this setting, several studies have identified endothelial dysfunction as a determinant factor towards coronary artery vasoconstriction and vasospasm, resulting in myocardial ischemia [36]. As abovementioned, inflammation has the possibility of impairing endothelial function throughout the reduction of endothelium-derived vasodilators bioavailability, thereby decreasing the expression of endothelial nitric oxide synthase (eNOS) and nitric oxide synthesis. Another potential mechanism is the cytokine-mediated imbalance of the autonomic nervous system. Specifically, the hypothalamic-pituitary-adrenal axis response to inflammation causes an upregulation of the sympathetic system leading to coronary vasoconstriction, affecting both macro and micro-circulation [37].

Although hyperglycemia in the context of MINOCA is still largely unexplored, it seems plausible that the same mechanisms described in obs-AMI may be valid in MINOCA as well. Supposedly, hyperglycemia can further precipitate the endothelial homeostasis and amplify the inflammatory process conferring an unbalanced vascular tone and a prothrombotic state, ultimately increasing the ischemic burden [38, 39].

\section{Infarct size and hyperglycemia in obstructive-AMI and MINOCA patients}

Hyperglycemic obs-AMI patients showed a larger infarct size than normoglycemic ones while in MINOCA no correlation was observed between admission glucose levels and the extent of myocardial damage, which was overall modest in such cases.

The link between hyperglycemia and large infarct size in the context of obs-AMI is well established, and our results are in line with previously published studies [40, 
MINOCA

NLR

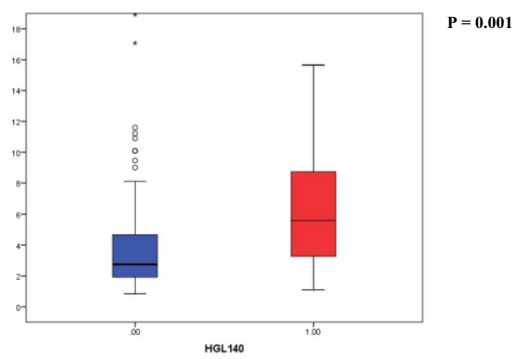

PLR

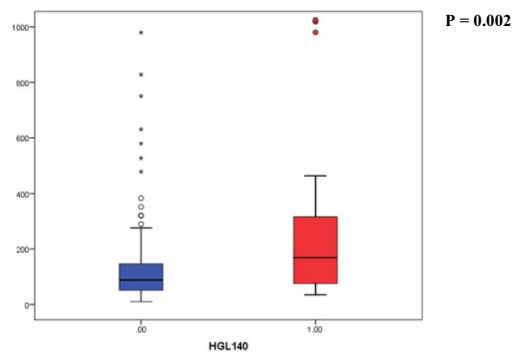

NPR

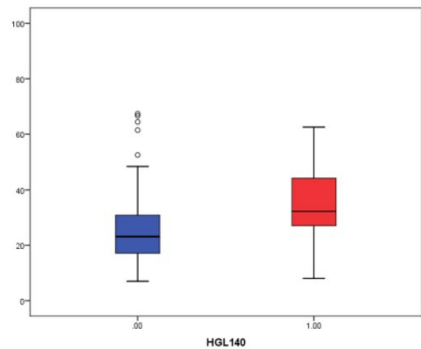

$\mathbf{P}<\mathbf{0 . 0 0 1}$
OBSTRUCTIVE-AMI

NLR

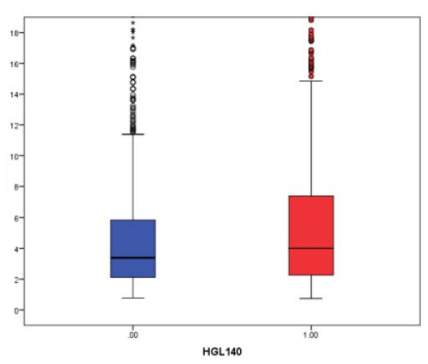

$\mathbf{P}<0.001$

PLR

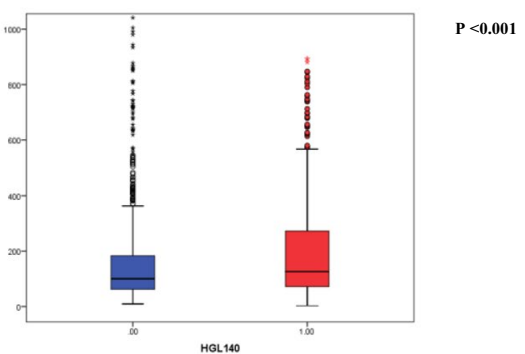

NPR

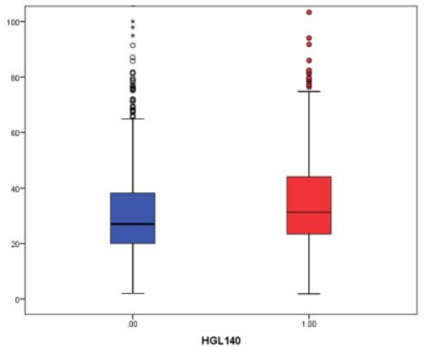

Fig. 2 Inflammatory markers in obstructive Acute Myocardial Infarction and Non-obstructive acute myocardial infarction. The blue colour denotes normoglycemic patients; the red colour represents hyperglycemic patients. AMI acute myocardial infarction, MINOCA non-obstructive acute myocardial infarction, NLR Neutrophil-to-lymphocyte ratio, NPR Neutrophil-to-platelet ratio, PLR Platelet-to-lymphocyte ratio

41]. In fact, several strategies have been adopted to assess the impact of admission hyperglycemia on the extent of myocardial damage, all leading toward the same conclusion $[42,43]$.

On the other hand, the impact of hyperglycemia on the magnitude of infarct size in MINOCA patients is still unexplored. Our findings suggest for the first time a negligible correlation between admission glucose levels and myocardial damage among MINOCA cases, who overall exhibited a limited infarct size, especially when compared to hyperglycemic obs-AMI subjects. The explanation to such results might be found in CMR studies specifically focused on the myocardial substrate of MINOCA. In particular, studies showed areas of myocardial oedema either associated with small necrotic regions with a typical patchy distribution or even without necrosis $[44,45]$. Since myocardial damage observed in our cohort was minimal, it is difficult to elucidate the actual impact of hyperglycemia in the still hazy world of MINOCA. Keeping in mind that endothelial dysfunction seems to be a key pathophysiological mechanism underlying MINOCA and it is known to be impaired by hyperglycemia as well, it appears plausible that an "hyperglycemic environment" can further alter the endothelial homeostasis and negatively affect the natural history of such patients, often characterized by heart failure with preserved ejection fraction. This hypothesis clearly requires future investigations in order to evaluate whether hyperglycemia may represent a prognostic risk factor for MINOCA subjects, regardless of a concomitant diabetes diagnosis [46-51].

\section{Study limitations}

Our study had several limitations. First of all, analyses were conducted on a relatively small sample size, 
especially regarding the MINOCA cohort. Second, not all laboratory parameters were available for each patient. Furthermore, it is not possible to clarify whether admission hyperglycemia has a direct relationship with infarct size and inflammatory markers or simply represents a marker of myocardial ischemia. In fact, the study does not establish causality between hyperglycemia and acute myocardial infarction by the nature of its cross-sectional design.

In patients with suspected DM, no definite rule-out criteria were adopted. However, not all patients can undergo an oral glucose tolerance test in the setting of AMI. Therefore, HbA1c could be a reasonable alternative in this clinical situation. Lastly, in our study we did not evaluate other inflammatory markers such as IL-6, TNF- $\alpha$, IL- 1 , and the soluble matricellular protein Cysteine-rich angiogenic inducer, which might reflect the inflammatory status more accurately. Taking into account that a correlation between such parameters and the indices adopted in our study was previously demonstrated [11-14, 47, 48], we chose to use common and widely available inflammatory markers for simplicity.

\section{Conclusion}

In patients with acute myocardial infarction, hyperglycemia was associated with a larger infarct size in obs-AMI while no differences were observed in MINOCA. Hyperglycemic obs-AMI cases presented elevated inflammatory markers both at admission and after $24 \mathrm{~h}$ whereas in MINOCA this data was evident only at the time of hospitalization, paralleling the modest myocardial damage detected in such patients. Our findings have pathophysiological and therapeutic implications, especially for obs-AMI subjects who can benefit from aggressive secondary therapies. Further prospective studies are needed to assess the prognostic role of hyperglycemia in the heterogenous MINOCA entity.

\section{Abbreviations \\ AMI: Acute myocardial infarction; T2DM: Type 2 diabetes mellitus; MACE: Major adverse cardiovascular events; aHGL: Admission high glucose levels; NLR: Neutrophil-to-lymphocyte ratio; PLR: Platelet-to-lymphocyte ratio; NPR: Neu- trophil-to-platelet ratio; MINOCA: Myocardial infarction with non-obstructive coronary arteries; Obs-AMl: Obstructive myocardial infarction; CAG: Coronary angiography; STEMI: ST-segment elevation acute myocardial infarction; LVEDV: Left-ventricular-end-diastolic-volume; LVEF: Left ventricular ejection fraction.}

\section{Acknowledgements}

None.

\section{Authors' contributions}

PP, LB and FD contributed conception and design of the study; FD, ST, MF, CC, $F A, A S, G$ and $M A$ organised the database; $L B$ and PR performed the statistical analysis; PP and LB wrote the first draft of the manuscript; CP and AF wrote sections of the manuscript. CP, AF, GC, CM and NG revised the article. All authors read and approved the final manuscript.
Funding

None.

\section{Availability of data and materials}

The datasets used and/or analysed during the current study are available from the corresponding author on reasonable request.

\section{Ethics approval and consent to participate}

Data were collected as part of an approved multicenter observational study called "AMIPE: Acute Myocardial Infarction, Prognostic and Therapeutic Evaluation" (ClinicalTrials.gov Identifier: NCT03883711). The present study was conducted according to the principles of the Declaration of Helsinki; all patients were informed about their participation in the registry and provided informed consent for the anonymous publication of scientific data.

\section{Consent for publication}

not applicable.

\section{Competing interests}

The authors declare that they have no competing interests.

\section{Author details}

${ }^{1}$ Unit of Cardiology, Department of Experimental, Diagnostic and Specialty Medicine-DIMES, University of Bologna, Via Giuseppe Massarenti 9 Bologna 40138, Italy. ${ }^{2}$ Division of Hygiene and Biostatistics, Department of Biomedical and Neuromotor Sciences, Alma Mater Studiorum, University of Bologna, Bologna, Italy. ${ }^{3}$ Unit of Cardiology, Maggiore Hospital, Bologna, Italy.

Received: 23 October 2020 Accepted: 20 January 2021

Published online: 02 February 2021

\section{References}

1. Capes SE, Hunt D, Malmberg K, Gerstein HC. Stress hyperglycaemia and increased risk of death after myocardial infarction in patients with and without diabetes: a systematic overview. Lancet. 2000;355:773-8.

2. Deedwania P, Kosiborod M, Barrett E, Ceriello A, Isley W, Mazzone T, Raskin P. Hyperglycemia and acute coronary syndrome: a scientific statement from the american heart association diabetes committee of the council on nutrition, physical activity, and metabolism. Circulation. 2008;117:1610-9.

3. Shah B, Amoroso NS, Sedlis SP. Hyperglycemia in nondiabetic patients presenting with acute myocardial infarction. Am J Med Sci. 2012:343:321-6.

4. Timmer JR, Hoekstra M, Nijsten MWN, van der Horst ICC, Ottervanger JP, Slingerland RJ, Dambrink JHE, Bilo HJG, Zijlstra F, van Hof AWJ. Prognostic value of admission glycosylated hemoglobin and glucose in nondiabetic patients with ST-segment-elevation myocardial infarction treated with percutaneous coronary intervention. Circulation. 2011;124:704-11.

5. Mi S, Su G, Yang H, Zhou Y, Tian L, Zhang T, Tao H. Comparison of inhospital glycemic variability and admission blood glucose in predicting short-term outcomes in non-diabetes patients with ST elevation myocardial infarction underwent percutaneous coronary intervention. Diabetol Metab Syndr. 2017;9:20.

6. Khalfallah M, Abdelmageed $\mathrm{R}$, Elgendy $\mathrm{E}$, Hafez $\mathrm{YM}$. Incidence, predictors and outcomes of stress hyperglycemia in patients with ST elevation myocardial infarction undergoing primary percutaneous coronary intervention. Diab Vasc Dis Res. 2020;17(1):1479164119883983.

7. Singh K, Hibbert B, Singh B, Carson K, Premaratne M, Le May M, Chong A-Y, Arstall M, So D. Meta-analysis of admission hyperglycaemia in acute myocardial infarction patients treated with primary angioplasty: a cause or a marker of mortality? Eur Heart J - Cardiovasc Pharmacother. 2015;1:220-8.

8. Angeli F, Reboldi G, Poltronieri C, Lazzari L, Sordi M, Garofoli M, Bartolini C, Verdecchia P. Hyperglycemia in acute coronary syndromes: from mechanisms to prognostic implications. Ther Adv Cardiovasc Dis. 2015;9:412-24.

9. Marik PE, Bellomo R. Stress hyperglycemia: an essential survival response. Crit Care. 2013;17(2):305. 
10. Kristiansen SB, Pælestik KB, Johnsen J, Jespersen NR, Pryds K, Hjortbak MV, Jensen RV, Bøtker HE. Impact of hyperglycemia on myocardial ischemiareperfusion susceptibility and ischemic preconditioning in hearts from rats with type 2 diabetes. Cardiovasc Diabetol. 2019;18:66.

11. Rock KL, Kono H. The inflammatory response to cell death. Annu Rev Pathol Mech Dis. 2008;3:99-126.

12. Chen C, Cong BL, Wang M, Abdullah M, Wang XL, Zhang YH, Xu SJ, Cui L. Neutrophil to lymphocyte ratio as a predictor of myocardial damage and cardiac dysfunction in acute coronary syndrome patients. Integr Med Res. 2018;7:192-9.

13. Somaschini A, Cornara S, Demarchi A, Mandurino-Mirizzi A, Fortuni F, Crimi G, Ferlini M, Camporotondo R, Gnecchi M, Visconti LO, De Ferrari GM. Neutrophil to platelet ratio: A novel prognostic biomarker in STelevation myocardial infarction patients undergoing primary percutaneous coronary intervention. Eur J Prev Cardiol. 2020;27(19):2338-40.

14. Li W, Liu Q, Tang Y. Platelet to lymphocyte ratio in the prediction of adverse outcomes after acute coronary syndrome: a meta-analysis. Sci Rep. 2017;7:40426.

15. Li H, Zhou Y, Ma Y, Han S, Zhou L. The prognostic value of the platelet-tolymphocyte ratio in acute coronary syndrome: a systematic review and meta-analysis. Kardiol Pol. 2017;75(7):666-73.

16. Thygesen K, Alpert JS, Jaffe AS, Chaitman BR, Bax JJ, Morrow DA, White HD, ESC Scientific Document Group, Thygesen K, Alpert JS, Jaffe AS, Chaitman BR, Bax JJ, Morrow DA, White HD, Mickley H, Crea F, Van de Werf F, Bucciarelli-Ducci C, Katus HA, Pinto FJ, Antman EM, Hamm CW, De Caterina R, Januzzi JL, Apple FS, Alonso Garcia MA, Underwood SR, Canty JM, Lyon AR, et al. Fourth universal definition of myocardial infarction (2018). Eur Heart J 2019;40:237-269.

17. Ibanez B, James S, Agewall S, Antunes MJ, Bucciarelli-Ducci C, Bueno H, Caforio ALP, Crea F, Goudevenos JA, Halvorsen S, Hindricks G, Kastrati A, Lenzen MJ, Prescott E, Roffi M, Valgimigli M, Varenhorst C, Vranckx P, Widimský P, ESC Scientific Document Group, Collet J-P, Kristensen SD, Aboyans V, Baumbach A, Bugiardini R, Coman IM, Delgado V, Fitzsimons D, Gaemperli O, Gershlick AH, et al. 2017 ESC Guidelines for the management of acute myocardial infarction in patients presenting with ST-segment elevation. Eur Heart J 2018;39:119-177.

18. Agewall S, Beltrame JF, Reynolds HR, Niessner A, Rosano G, Caforio AL, De Caterina R, Zimarino M, Roffi M, Kjeldsen K, Atar D, Kaski JC, Sechtem U, Tornvall P; WG on Cardiovascular Pharmacotherapy. ESC working group position paper on myocardial infarction with non-obstructive coronary arteries. Eur Heart J. 2017;38(3):143-153.

19. Paolisso P, Bergamaschi L, Saturi G, D'Angelo EC, Magnani I, Toniolo S, Stefanizzi A, Rinaldi A, Bartoli L, Angeli F, Donati F, Rucci P, Mattioli AV, Taglieri N, Pizzi C, Galiè N. Secondary prevention medical therapy and outcomes in patients with myocardial infarction with non-obstructive coronary artery disease. Front Pharmacol. 2020;10:1606.

20. Lang RM, Badano LP, Mor-Avi V, Afilalo J, Armstrong A, Ernande L, Flachskampf FA, Foster E, Goldstein SA, Kuznetsova T, Lancellotti P, Muraru D, Picard MH, Rietzschel ER, Rudski L, Spencer KT, Tsang W, Voigt J-U. Recommendations for cardiac chamber quantification by echocardiography in adults: an update from the american society of echocardiography and the european association of cardiovascular imaging. Eur Heart J Cardiovasc Imaging. 2015;16:233-71.

21. American Diabetes Association. 2. Classification and Diagnosis of Diabetes: Standards of Medical Care in Diabetes-2019. Diabetes Care 2019;42:S13-S28.

22. Marfella R, Siniscalchi M, Esposito K, Sellitto A, de Fanis U, Romano C, Portoghese M, Siciliano S, Nappo F, Sasso FC, Mininni N, Cacciapuoti F, Lucivero G, Giunta R, Verza M, Giugliano D. Effects of stress hyperglycemia on acute myocardial infarction: role of inflammatory immune process in functional cardiac outcome. Diabetes Care. 2003;26:3129-35.

23. Esper RJ, Nordaby RA. Cardiovascular events, diabetes and guidelines: the virtue of simplicity. Cardiovasc Diabetol. 2019;18:42.

24. Wong ND, Toth PP, Amsterdam EA; American Society for Preventive Cardiology Most important advances in preventive cardiology during this past decade: Viewpoint from the American Society for Preventive Cardiology. Trends Cardiovasc Med. 2019:S1050-1738(19)30163-X.

25. Galkina E, Ley K. Immune and inflammatory mechanisms of atherosclerosis. Annu Rev Immunol. 2009;27:165-97.
26. Centurión OA. Serum biomarkers and source of inflammation in acute coronary syndromes and percutaneous coronary interventions. Cardiovasc Revasc Med. 2016;17:119-28.

27. Kitano D, Takayama T, Nagashima K, Akabane M, Okubo K, Hiro T, Hirayama A. A comparative study of time-specific oxidative stress after acute myocardial infarction in patients with and without diabetes mellitus. BMC Cardiovasc Disord. 2016;16:102.

28. Giacco F, Brownlee M. Oxidative Stress and Diabetic Complications. Circ Res. 2010;107:1058-70.

29. Lin B, Ginsberg MD, Busto R, Li L. Hyperglycemia triggers massive neutrophil deposition in brain following transient ischemia in rats. Neurosci Lett. 2000;278:1-4.

30. Borst SE. The role of TNF-a in insulin resistance. Endocrine. 2004;23(2-3):177-82

31. Jager J, Grémeaux T, Cormont M, Le Marchand-Brustel Y, Tanti J-F. Interleukin-1 $\beta$-induced insulin resistance in adipocytes through downregulation of insulin receptor substrate-1 expression. Endocrinology. 2007;148:241-51

32. Barnes TM, Otero YF, Elliott AD, Locke AD, Malabanan CM, Coldren AG, Brissova M, Piston DW, McGuinness OP. Interleukin-6 amplifies glucagon secretion: coordinated control via the brain and pancreas. Am J PhysiolEndocrinol Metab. 2014;307:E896-905.

33. Liu J, Wang S, Cui C, Cai H, Sun R, Pan W, Fang S, Yu B. The association between glucose-related variables and plaque morphology in patients with ST-segment elevated myocardial infarction. Cardiovasc Diabetol. 2020;19:109.

34. de Mulder M, Cornel J-H, van der Ploeg T, Boersma E, Umans VA. Elevated admission glucose is associated with increased long-term mortality in myocardial infarction patients, irrespective of the initially applied reperfusion strategy. Am Heart J. 2010;160:412-9.

35. Tamis-Holland JE, Jneid H, Reynolds HR, Agewall S, Brilakis ES, Brown TM, Lerman A, Cushman M, Kumbhani DJ, Arslanian-Engoren C, Bolger AF, Beltrame JF; American Heart Association Interventional Cardiovascular Care Committee of the Council on Clinical Cardiology; Council on Cardiovascular and Stroke Nursing; Council on Epidemiology and Prevention; and Council on Quality of Care and Outcomes Research. Contemporary Diagnosis and Management of Patients With Myocardial Infarction in the Absence of Obstructive Coronary Artery Disease: A Scientific Statement From the American Heart Association. Circulation. 2019;139(18):e891-e908.

36. Beijk MA, Vlastra WV, Delewi R, van de Hoef TP, Boekholdt SM, Sjauw KD, Piek JJ. Myocardial infarction with non-obstructive coronary arteries: a focus on vasospastic angina. Neth Heart J. 2019;27:237-45.

37. Burford N, Webster N, Cruz-Topete D. Hypothalamic-pituitary-adrenal axis modulation of glucocorticoids in the cardiovascular system. Int J Mol Sci. 2017:18:2150.

38. Sara JD, Taher R, Kolluri N, Vella A, Lerman LO, Lerman A. Coronary microvascular dysfunction is associated with poor glycemic control amongst female diabetics with chest pain and non-obstructive coronary artery disease. Cardiovasc Diabetol. 2019;18(1):22.

39. Sardu C, Paolisso P, Sacra C, Mauro C, Minicucci F, Portoghese M, Rizzo MR, Barbieri M, Sasso FC, D'Onofrio N, Balestrieri ML, Calabrò P, Paolisso G, Marfella R. Effects of metformin therapy on coronary endothelial dysfunction in patients with prediabetes with stable angina and nonobstructive coronary artery stenosis: The CODYCE Multicenter Prospective Study. Diabetes Care. 2019;42(10):1946-55.

40. Eitel I, Hintze S, de Waha S, Fuernau G, Lurz P, Desch S, Schuler G, Thiele H. Prognostic impact of hyperglycemia in nondiabetic and diabetic patients With ST-elevation myocardial infarction: insights from contrast-enhanced magnetic resonance imaging. Circ Cardiovasc Imaging. 2012;5:708-18.

41. Zhu Y, Liu K, Meng S, Jia R, Lei X, Chen M, Zou K, Zhu H, Jin Z. Augmented glycaemic gap is a marker for an increased risk of post-infarct left ventricular systolic dysfunction. Cardiovasc Diabetol. 2020;19:101.

42. Lonborg J, Vejlstrup N, Kelbaek H, Nepper-Christensen L, Jorgensen E, Helqvist S, Holmvang L, Saunamaki K, Botker HE, Kim WY, Clemmensen P, Treiman M, Engstrom T. Impact of acute hyperglycemia on myocardial infarct size, area at risk, and salvage in patients With STEMI and the association with exenatide treatment: results from a randomized study. Diabetes. 2014;63:2474-85.

43. Cruz-Gonzalez I, Chia S, Raffel OC, Sanchez-Ledesma M, Senatore F, Wackers FJ, Nathan DM, Jang I-K. Hyperglycemia on admission predicts larger 
infarct size in patients undergoing percutaneous coronary intervention for acute ST-segment elevation myocardial infarction. Diabetes Res Clin Pract. 2010;88:97-102.

44. Baccouche H, Mahrholdt H, Meinhardt G, Merher R, Voehringer M, Hill S, Klingel K, Kandolf R, Sechtem U, Yilmaz A. Diagnostic synergy of noninvasive cardiovascular magnetic resonance and invasive endomyocardial biopsy in troponin-positive patients without coronary artery disease. Eur Heart J. 2009;30(23):2869-79.

45. Collste $O$, Sörensson P, Frick M, Agewall S, Daniel M, Henareh L, Ekenbäck C, Eurenius L, Guiron C, Jernberg T, Hofman-Bang C, Malmqvist K, Nagy E, Arheden $\mathrm{H}$, Tornvall P. Myocardial infarction with normal coronary arteries is common and associated with normal findings on cardiovascular magnetic resonance imaging: results from the Stockholm Myocardial Infarction with Normal Coronaries study. J Intern Med. 2013;273(2):189-96.

46. Kosmas N, Manolis AS, Dagres N, Iliodromitis EK. Myocardial infarction or acute coronary syndrome with non-obstructive coronary arteries and sudden cardiac death: a missing connection. EP Eur. 2020;22:1303-10.

47. Rossello X, Bueno H, Pocock SJ, Van de Werf F, Danchin N, Annemans L, Medina J, Zeymer U. Predictors of all-cause mortality and ischemic events within and beyond 1 year after an acute coronary syndrome: Results from the EPICOR registry. Clin Cardiol. 2019;42:111-9.

48. Pocock SJ, Huo Y, Van de Werf F, Newsome S, Chin CT, Vega AM, Medina J, Bueno H. Predicting two-year mortality from discharge after acute coronary syndrome: An internationally-based risk score. Eur Heart J Acute Cardiovasc Care. 2019;8:727-37.

49. Turkmen K, Guney I, Yerlikaya FH, Tonbul HZ. The relationship between neutrophil-to-lymphocyte ratio and inflammation in end-stage renal disease patients. Ren Fail. 2012;34:155-9.

50. Winzap P, Davies A, Klingenberg R, Obeid S, Roffi M, Mach F, Räber L, Windecker S, Templin C, Nietlispach F, Nanchen D, Gencer B, Muller O, Matter CM, von Eckardstein A, Lüscher TF. Diabetes and baseline glucose are associated with inflammation, left ventricular function and shortand long-term outcome in acute coronary syndromes: role of the novel biomarker Cyr 61. Cardiovasc Diabetol. 2019;18:142.

51. Pizzi C, Xhyheri B, Costa GM, Faustino M, Flacco ME, Gualano MR, Fragassi G, Grigioni F, Manzoli L. Nonobstructive Versus Obstructive Coronary Artery Disease in Acute Coronary Syndrome: A Meta-Analysis. J Am Heart Assoc. 2016;5(12):004185

\section{Publisher's Note}

Springer Nature remains neutral with regard to jurisdictional claims in published maps and institutional affiliations.
Ready to submit your research? Choose BMC and benefit from:

- fast, convenient online submission

- thorough peer review by experienced researchers in your field

- rapid publication on acceptance

- support for research data, including large and complex data types

- gold Open Access which fosters wider collaboration and increased citations

- maximum visibility for your research: over $100 \mathrm{M}$ website views per year

At BMC, research is always in progress.

Learn more biomedcentral.com/submissions 